\title{
Interferometric methods for static and dynamic characterizations of micromembranes for sensing functions
}

\author{
L. Salbut ${ }^{1}$, J. Kacperski ${ }^{1}$, A. Styk ${ }^{1}$, M. Jozwik ${ }^{1,2}$, C. Gorecki ${ }^{2}$, H. Urey ${ }^{3}$, \\ A. Jacobelli ${ }^{4}$, T. Dean ${ }^{4}$ \\ ${ }^{1}$ Warsaw University of Technology, Poland \\ ${ }^{2}$ Université de Franche-Comté, France \\ ${ }^{3}$ Koc University, Turkey \\ ${ }^{4}$ Thales Research and Technology, France
}

\begin{abstract}
We present a methodology for static and dynamic testing of mechanical properties of microelements. The measurement path includes temporal phase shifting interferometry for quantitative static shape elements analysis. This is followed by determination of the resonance frequency by means of modified time average interferometry and transient amplitude and phase maps of vibrating micromembrane capturing and evaluation by phase shifting stroboscopic interferometry. Proper application of combination of these methods allows for quick and accurate analysis of micromembranes and optimization of their manufacturing conditions.
\end{abstract}

Keywords: microelements, MEMS/MOEMS, optical metrology, microinterferometry, vibration analysis

\section{INTRODUCTION}

The objective of this work is to develop the new testability procedures for characterization of the mechanical behavior of Micro-Electro-Mechanical systems (MEMS), specifically active micromembranes, covering their performance during all stages of manufacturing from the design to the operation of finished product. High sensitive and accurate measurements are required for automatic static membrane shape determination and monitoring of value and phase of out-of plane displacement at chosen frequencies and stages of vibration. The measurement approach is based on conventional twobeam interferometry [1] coupled with time average [2,3] and stroboscopic interferometry [4] which support global and local analysis of vibrating micromembranes.

Due to applying a convenient method of automatic shape determination the measurements were performed for all membranes manufactured at the silicon wafer providing an excellent data base for extended analysis focused on optimization of technological parameters of membranes manufacturing.

\section{OBJECTS UNDER TEST}

The measured objects were fabricated by THALES Research \& Technology and Dépt. LOPMD - Université de Franche-Comté on SOI wafers (silicon on insulator) (Fig.1a). The object selected for testing included square micromembranes with dimensions $0.45 \times 0.45 \mathrm{~mm}^{2}$ and $1.35 \times 1.35 \mathrm{~mm}^{2}$ (Fig. 1b). 28 membranes of each dimension were produced at 4 inch silicon wafer as shown in Fig. 1b. Due to the technological process, which includes introducing $\mathrm{SiO}_{\mathrm{x}} \mathrm{N}_{\mathrm{y}}$ and PZT layers, the membranes suffer a departure from the flat shape. Depending on the membrane localization within the wafer the initial deformations differ significantly.

The membranes were tested under static and dynamic conditions. They were activated by applying 5 or $10 \mathrm{~V}$ voltage at their electrodes. 
a)
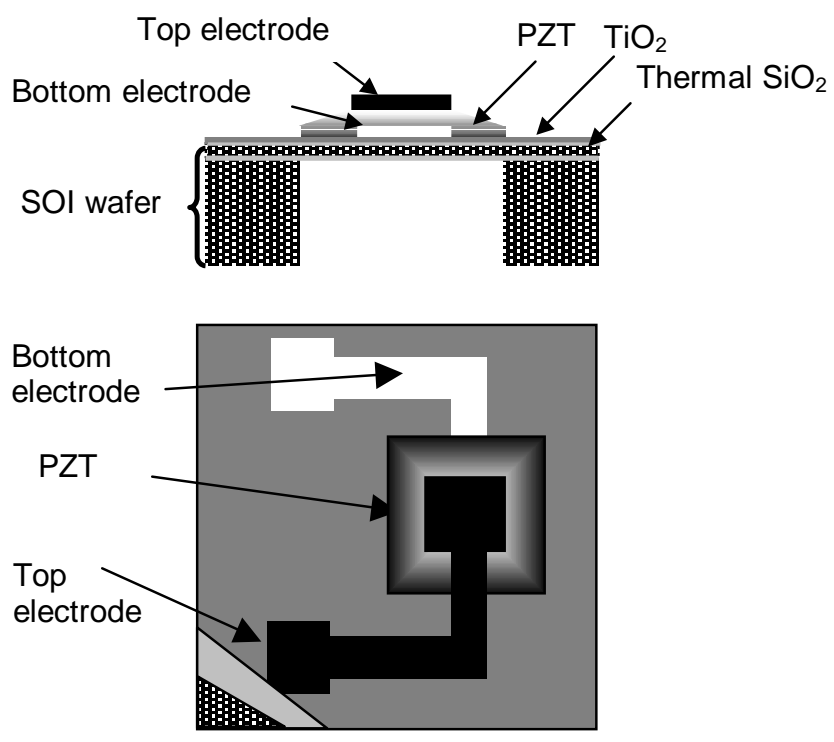

b)

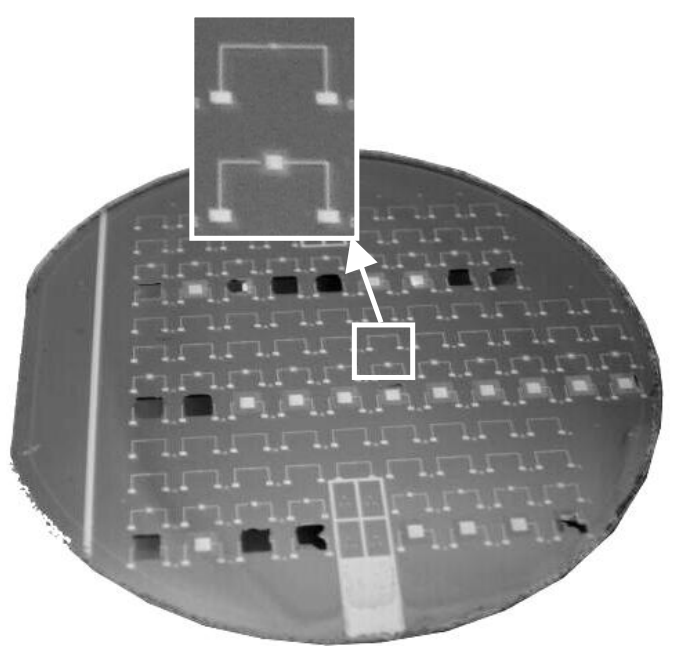

Fig.1. Micromembranes under test: a) scheme of design and b) $1.35 \times 1.35 \mathrm{~mm}^{2}$ and $0.45 \times 0.45 \mathrm{~mm}^{2}$ membranes and wafer.

\section{MEASUREMENT TECHNIQUES}

The general concept of measurement is shown in Fig.2. In the first stage the initial shape of micromembrane is determined by conventional two-beam phase shifting interferometry.

The intensity distribution in the two-beam interferogram can be described as:

$$
I_{\text {stat }}(x, y)=K\left\{1+C_{\text {stat }} \cos \varphi_{\text {stat }}(x, y)\right\},
$$

where:

$K=a^{2}(x, y)+b^{2}(x, y)$ denotes the spatial distribution of the sum of intensities of two beams (the interferogram bias), the amplitudes of beams are $a(x, y)$ and $b(x, y)$,

$C_{\text {stat }}=\frac{2 a(x, y) b(x, y)}{a^{2}(x, y)+b^{2}(x, y)}$ denotes the modulation depth (contrast) of interference fringes,

$\varphi_{\text {stat }}(x, y)=(2 \pi / \lambda) \cdot O P D(x, y)$, where $O P D(x, y)$ is the optical path difference between the reference beam and object beam wave fronts; it includes the influence of the mean position and non-flatness of the object surface, $\lambda$ is the light wavelength.

The index ,stat" refers to the case of stationary (nonvibrating) object under test. For interferogram evaluation the fiveframe phase shifting method is applied and the shape of the object is obtained in the form of $w_{0}(x, y)$ map.

The second stage of measurement requires measurement of resonance mode shapes (amplitude and phase distributions). This is performed in several steps. At first the accurate determination of resonance frequencies is required. It can be achieved by using external point measurement devices, however we propose to apply time average interferometry (TAI). The contrast fringe pattern at resonance frequencies is modulated by zero order Bessel function and it can be shown that in the case of a sinusoidal object vibration the interferogram intensity distribution can be written as:

$$
I_{\text {vibr }}(x, y)=K\left\{1+C_{\text {stat }} J_{0}\left(\frac{4 \pi}{\lambda} a_{0}\right) \cos \varphi_{\text {vibr }}(x, y)\right\}
$$

where: $J_{0}$ - zero order Bessel function, $a_{0}$ - vibration amplitude. The sensitivity factor $(4 \pi / \lambda)$ appearing in the argument of the Bessel function relates to the experimental setup with the illuminating beam and observation directions coinciding 
with the normal to the element under test (maximum sensitivity configuration). The phase $\varphi_{\text {vibr }}(x, y)$ has been differentiated from $\varphi_{\text {stat }}(x, y)$ allowing possible change of the mean position of the object under test between static and vibration states.

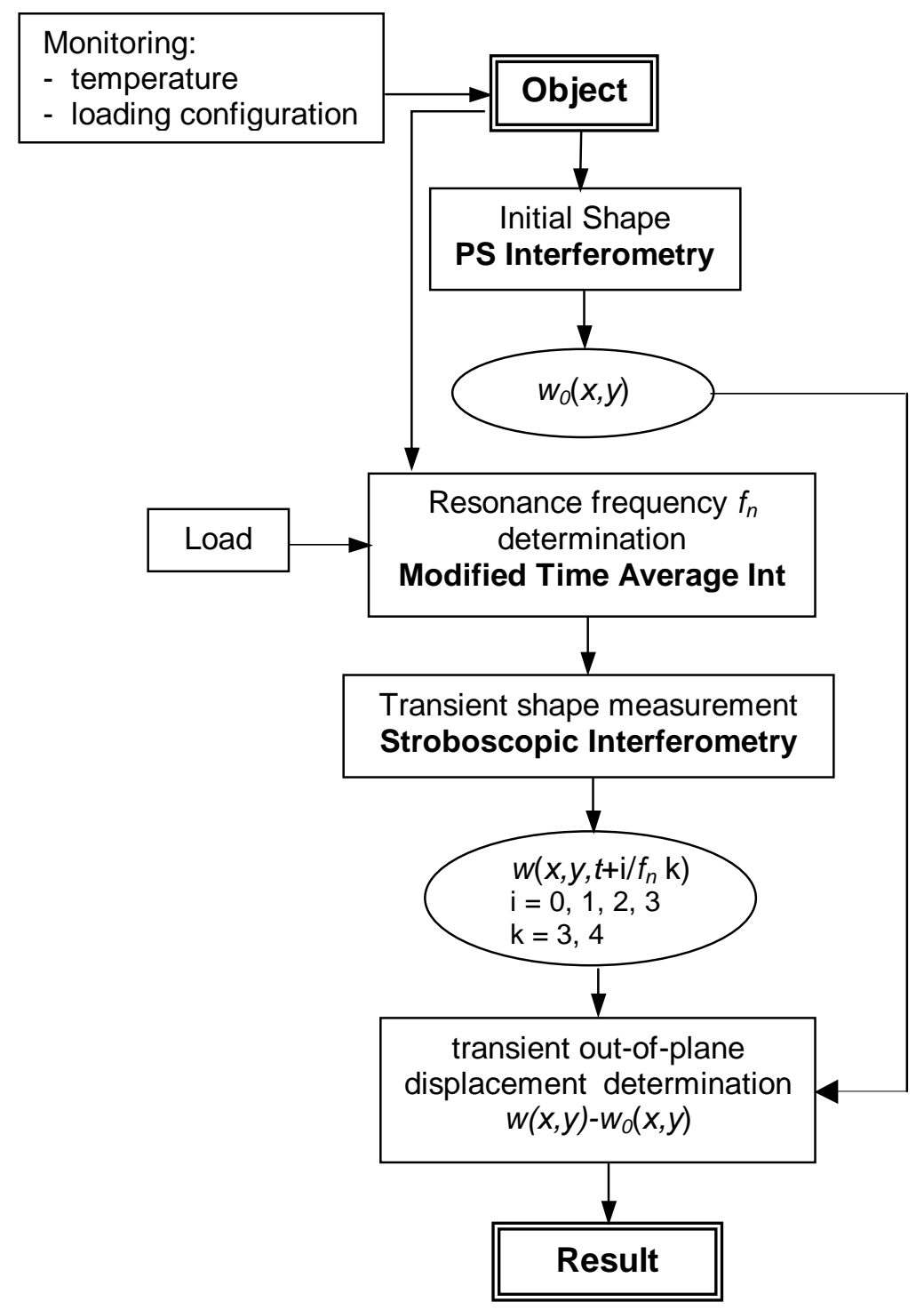

Fig. 2: The scheme of measurement sequence used for active micromembrane characterization.

As seen in Fig. 3a the time average interferometry provides interferograms with low contrast Bessel fringes superimposed on the fringes which contain information about shape of an element. This data has to be enhanced significantly. It is performed by four-frame phase stepping method proposed by Patorski et al. [3]. 


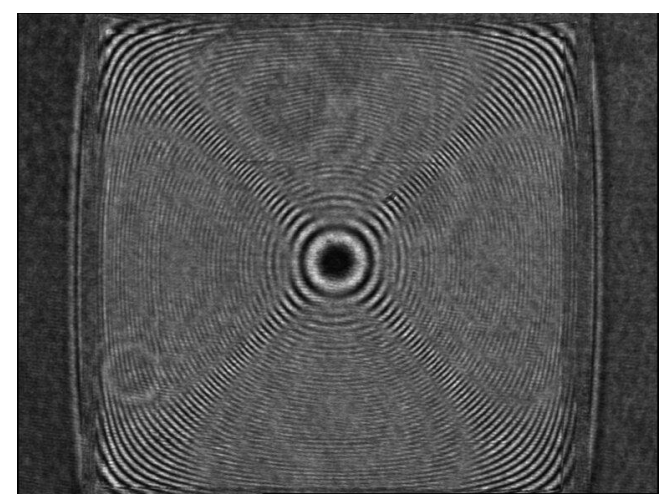

b)

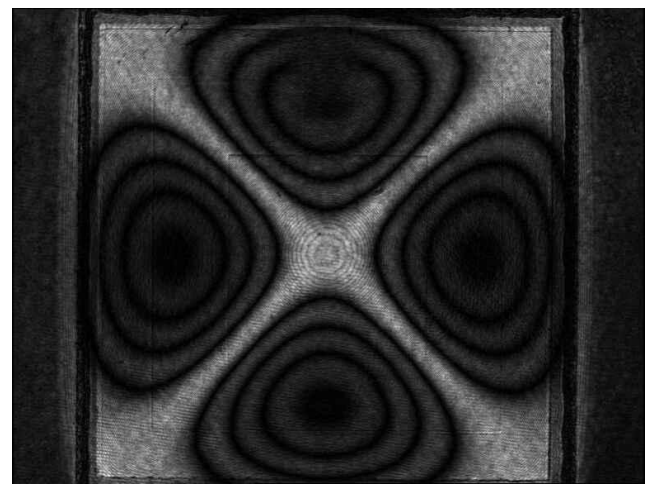

Fig. 3: Time-average interferogram obtained for a square membrane at $v_{0}=172.8 \mathrm{kHz}$ : a) direct, and b) enhanced.

This approach involves recording four interferograms mutually phase shifted by $\pi / 2$ for the static and resonance mode states and performing arithmetic operation at these intensities. As the result we obtain:

a) for the static object:

$$
\left(I_{1}-I_{3}\right)^{2}+\left(I_{2}-I_{4}\right)^{2}=4 K^{2} C_{\text {stat }}^{2}
$$

b) for the vibrating object:

$$
\left(I_{1}-I_{3}\right)^{2}+\left(I_{2}-I_{4}\right)^{2}=4 K^{2} C_{s t a t}^{2} J_{0}^{2}\left(\frac{4 \pi}{\lambda} a_{0}\right)
$$

Dividing the results $\left(I_{1}-I_{3}\right)^{2}+\left(I_{2}-I_{4}\right)^{2}$ obtained for the vibrating and static object states, Eqs. (3) and (4), we get enhanced, clear image of Bessel fringes (Fig. 3b):

$$
\frac{4 K^{2} C_{\text {stat }}^{2} J_{0}^{2}\left(\frac{4 \pi}{\lambda} a_{0}\right)}{4 K^{2} C_{\text {stat }}^{2}}=J_{0}^{2}\left(\frac{4 \pi}{\lambda} a_{0}\right)
$$

However the software supporting classical interferometric techniques has to be significantly changed in order to properly determine full field vibration amplitude distribution from Bessel fringes. Also the sign of amplitude (its phase) cannot be determined from Bessel fringes. Therefore this procedure was extensively used for precise determination of resonance frequencies only.

For vibration modes profiling by stroboscopic interferometry [4], a light pulse of width $\delta$, synchronized with the vibration excitation signal (see Fig.4) but with an adjustable delay time $t_{m}$ is used to freeze the object vibration at any time of the vibration cycle. The light detected by the camera is integrated over a time $T_{D}$ related to the video rate, which for micromechanical devices is always very large with respect to the period $\mathrm{T}$ of the vibration.

a)

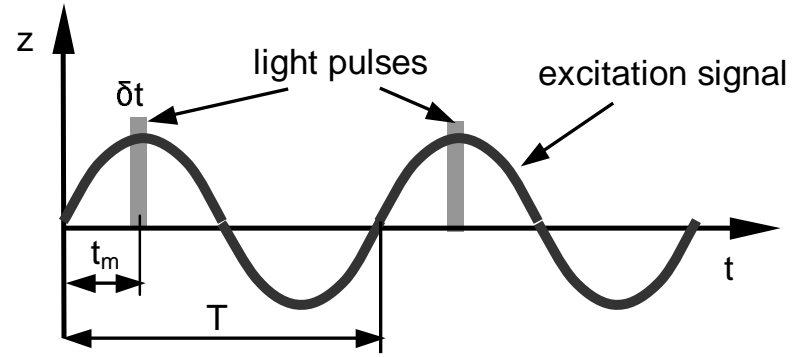

b)

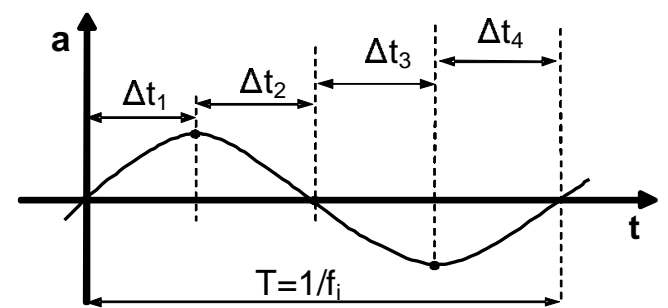

$\Delta \mathrm{t}_{\mathrm{i}}=\mathrm{i} / f_{n} \mathrm{k}$ where $\mathrm{i}=1,2,3,4$ and $\mathrm{k}=4$

Fig. 4: The principle of stroboscopic technique: a) synchronization between excitation signal and light pulses and b) step measurements within a single vibration period. 
The captured intensity can be expressed in the following form:

$$
I(x, y)=K\left\{1+C_{s t a t} A\left(a_{0}, \delta t\right) \cos \left[\varphi_{v i b r}(x, y)+\frac{4 \pi}{\lambda} a(x, y)\right]\right\}
$$

where:

$K$ and $C_{\text {stat }}$ are the interferogram bias and contrast for static state, respectively,

$\varphi_{v i b r}$ is the optical path difference between reference and object beams for mean position of the vibrating object under test,

$a(x, y)$ is the function describing vibration mode shape,

$a_{0}$ is maximal object amplitude,

$A\left(a_{0}, \delta t\right)$ is the attenuation function described as:

$$
\mathrm{A}\left(\mathrm{a}_{0}, \mathrm{~T}\right)=\left[\mathrm{J}_{0}\left(\frac{4 \pi}{\lambda} \mathrm{a}_{0}\right)+2 \sum_{\mathrm{n}=1}^{\infty}(\mathrm{i})^{\mathrm{n}} \sin \mathrm{c}\left(\frac{\mathrm{n} \omega \mathrm{T}}{2}\right) \mathrm{J}_{\mathrm{n}}\left(\frac{4 \pi}{\lambda} \mathrm{a}_{0}\right)\right]^{2}
$$

For shorter light pulse the larger vibration amplitude can be determined, so application of stroboscopic interferometry is limited by frequency of diode laser driver used for measurement. In our case the objects vibrated with frequency a few kilohertz will be tested. The transient shape of a membrane is measured for its predetermined positions within a single vibration period (see Fig. 4b).

\section{MEASUREMENT SYSTEM}

Scheme of the measurement stand is shown in Fig. 5. It is Twyman-Green interferometer integrated with optical microscope and a variety of supporting devices for object manipulation and loading and for synchronization between object loading system and pulse light source controller.

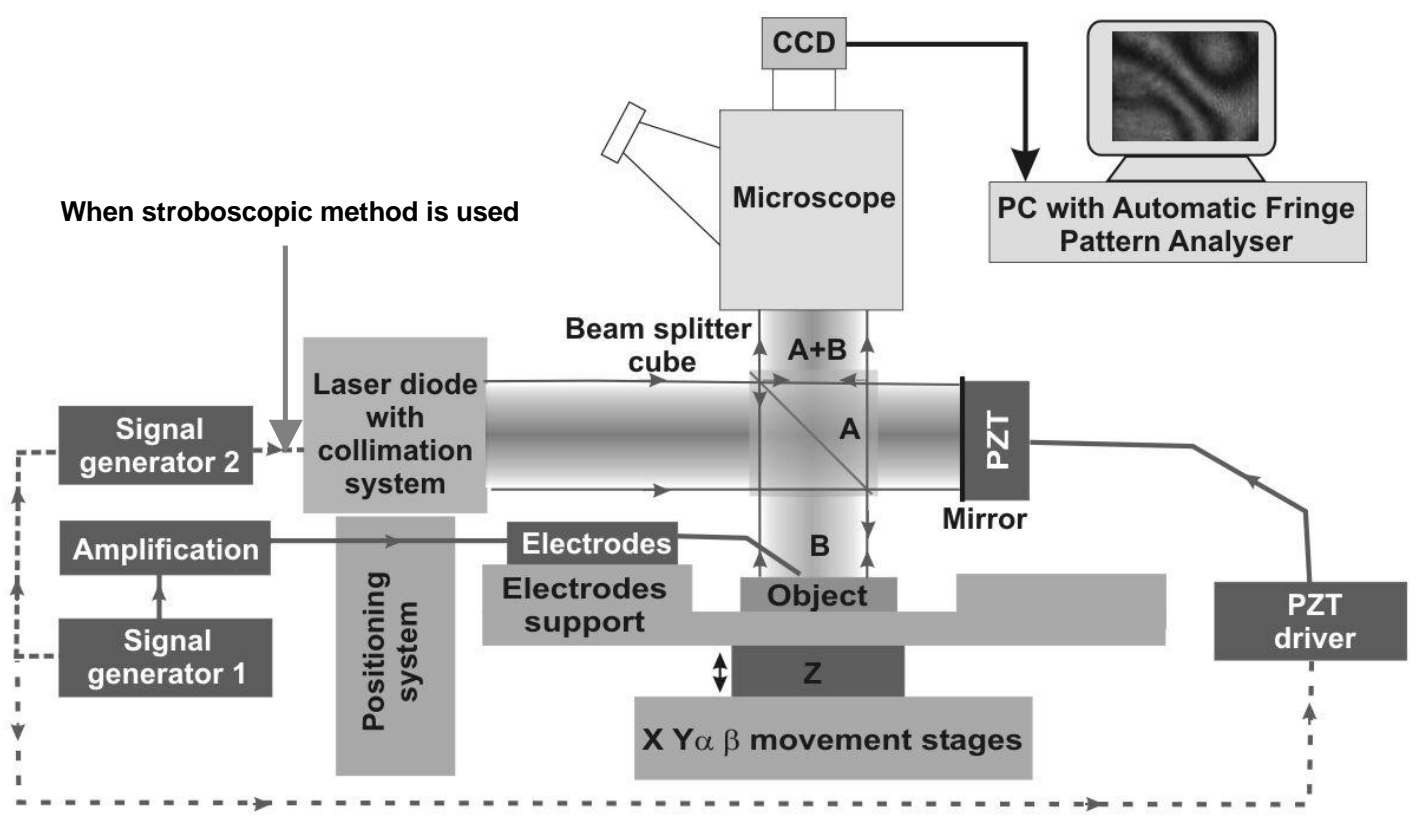

Fig. 5: Scheme of the measurement stand for a) static and time-average interferometry based measurements and its modification for b) stroboscopic interferometry. 


\section{MEASUREMENT RESULTS}

\subsection{Static measurements}

The results of measurements of static shape of micromembranes were performed in two stages. At first the individual shape maps for each membrane at the wafer were obtained (see Fig. 6). Secondly the statistic analysis of the membranes parameters distribution at the silicon wafer was performed.

a)

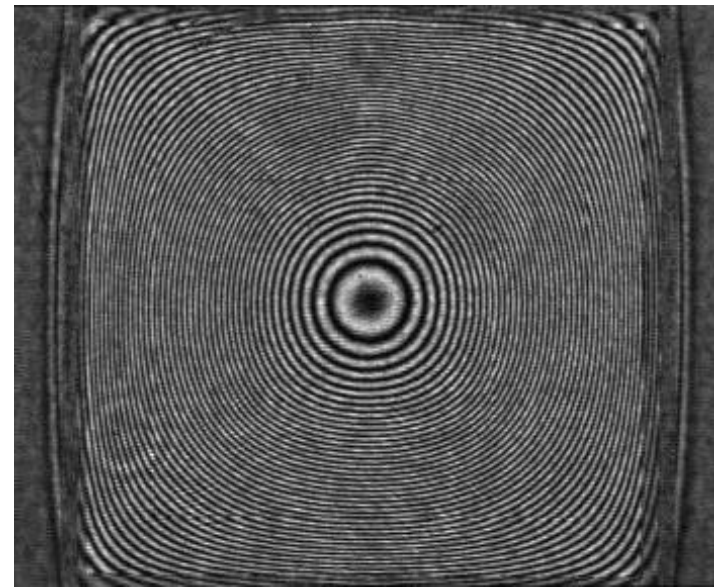

b)

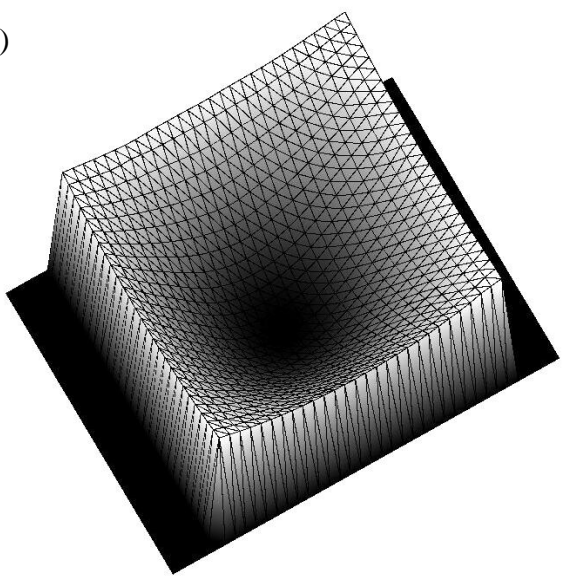

Fig. 6: Example of the static interferogram (a) and corresponding 3D shape representations of $1.35 \times 1.35 \mathrm{~mm}^{2}$ membrane (b).

In the case of $0.45 \times 0.45 \mathrm{~mm}^{2}$ membranes measurement we assumed centre point of each membrane as a reference. Deformation value in this point (reference point) is assumed to be zero. That approach allowed us to compare values of initial deformation of the membranes. For better comparison we calculated value of average deformation of each membrane. That value equals arithmetic average of deformation values in points placed at $0.08 \mathrm{~mm}$ distance from centre of the membrane in horizontal and vertical direction. Comparison of average deformation of those membranes (numbered as shown in Fig .7) is shown in Fig. 8 and Tab. 1. We can see that membranes placed nearer to the edge of wafer have bigger values of deformation.

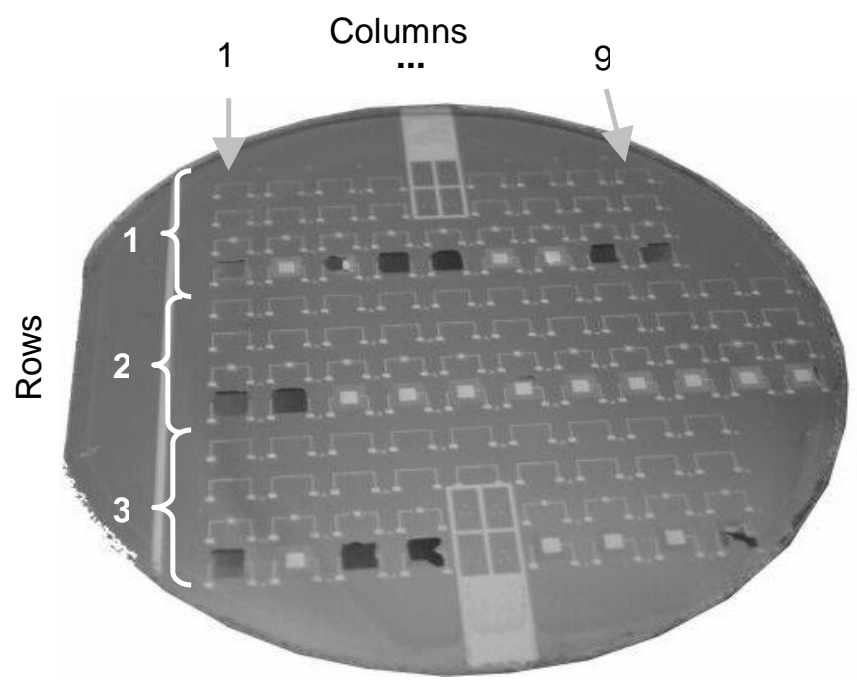

Fig. 7: The way of numbering of the membranes. 


\begin{tabular}{|l|c|c|c|c|c|c|c|c|c|c|c|}
\cline { 2 - 12 } \multicolumn{1}{c|}{} & $\mathbf{1}$ & $\mathbf{2}$ & $\mathbf{3}$ & $\mathbf{4}$ & $\mathbf{5}$ & $\mathbf{6}$ & $\mathbf{7}$ & $\mathbf{8}$ & $\mathbf{9}$ & $\mathbf{1 0}$ & $\mathbf{1 1}$ \\
\hline $\mathbf{1}$ & 517 & 374 & 239 & 197 & 176 & 110 & 169 & 514 & 277 & - & - \\
\hline $\mathbf{2}$ & 295 & 253 & 327 & 222 & 199 & 236 & 216 & 307 & 386 & 455 & 642 \\
\hline $\mathbf{3}$ & 435 & 373 & 330 & 291 & - & 243 & 293 & 448 & 399 & - & - \\
\hline
\end{tabular}

Tab. 1: Average initial deformation values of the membranes [nm].

(Highlighted membranes were dynamically tested with the use of the stroboscopic method)

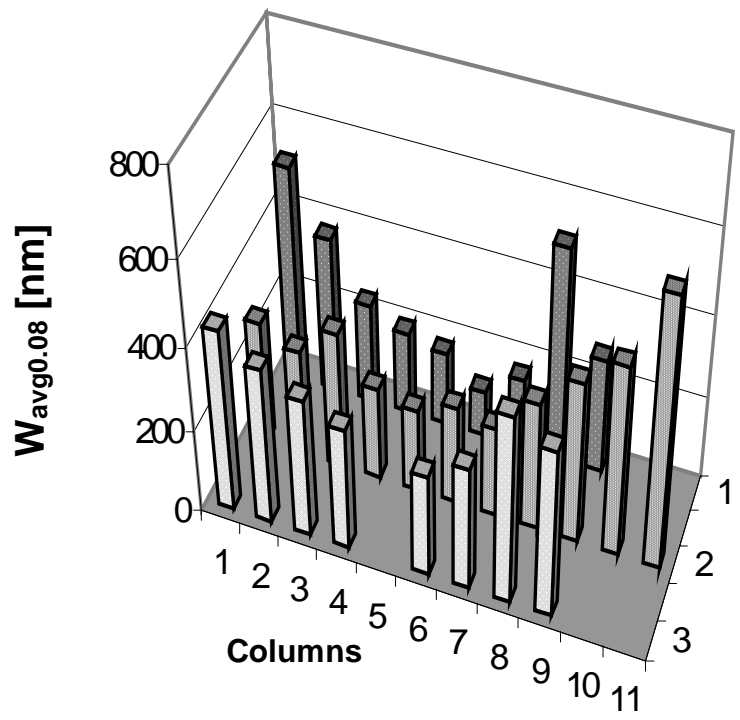

Rows

Fig. 8: Analysis of a set of micromembranes $0.45 \times 0.45 \mathrm{~mm}^{2}$ at wafer; $\mathrm{w}_{\mathrm{avg} 0.08}$ - average initial deformation values.

\subsection{Resonance frequency determination}

The modified time average interferometry method was applied for determination of the first three resonance frequencies of the micromembranes. The exemplary enhanced Bessel fringes obtained for excitation of the membrane with dimension $1.35 \times 1.35 \mathrm{~mm}^{2}$ are shown in Fig. 9 .

a)

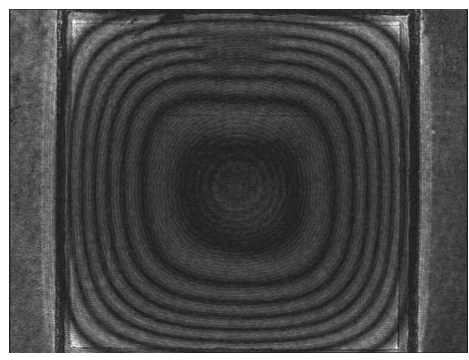

b)

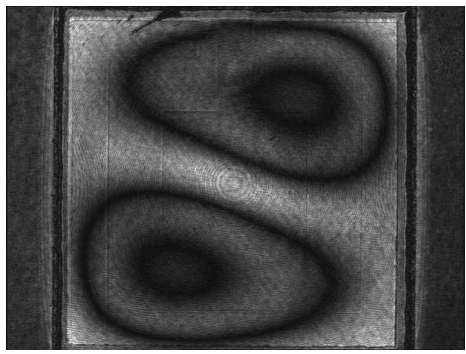

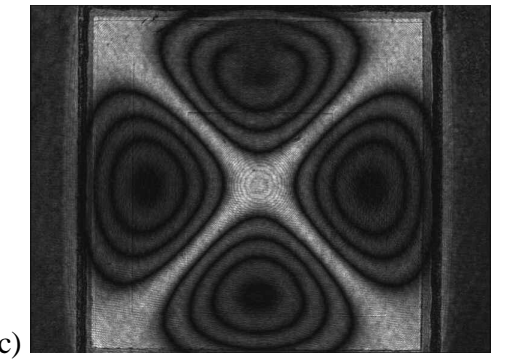

c)
Fig. 9: Images obtained for $\mathrm{U}=10 \mathrm{~V}$ at different resonance frequencies: a) $91.1 \mathrm{kHz}$; b) $107.4 \mathrm{kHz}$; ) $172.8 \mathrm{kHz}$.

For membrane with dimension $0.45 \times 0.45 \mathrm{~mm}^{2}$ the first two resonance frequencies were determined as $128 \mathrm{kHz}$ and $385 \mathrm{kHz}$.

\subsection{Transient membrane deformation measurements}

Extended series of measurements of micromembranes were performed. Below we present the data obtained for the micromembrane with dimension $0.45 \times 0.45 \mathrm{~mm}^{2}$. The data registered include interferograms for the $1^{\text {st }}$ and $2^{\text {nd }}$ resonance frequencies. 
For each frequency and value of excitation signal amplitude 4 interferograms with spatial carrier: for $0,90,180$ and $270^{\circ}$ of phase difference between excitation signal and diode controlling signal were registered.

I a)
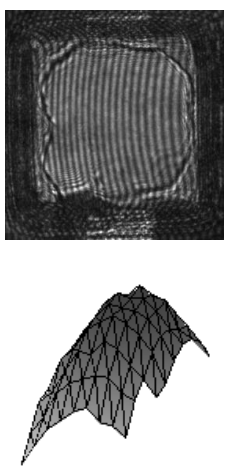

II a)
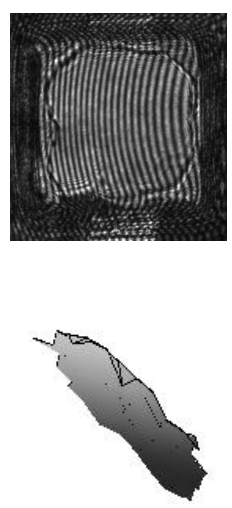

b)

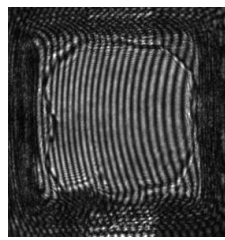

b)
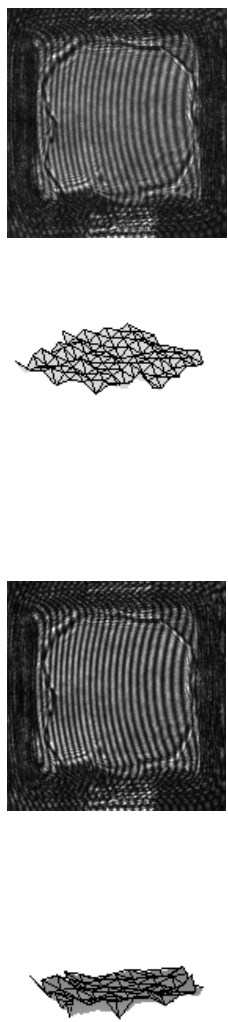

c)

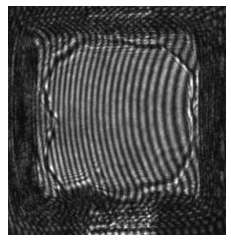

d)
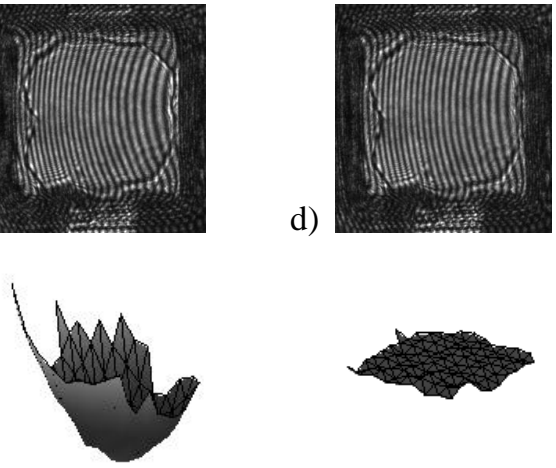

c)

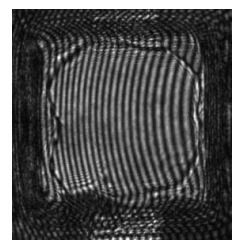

d)
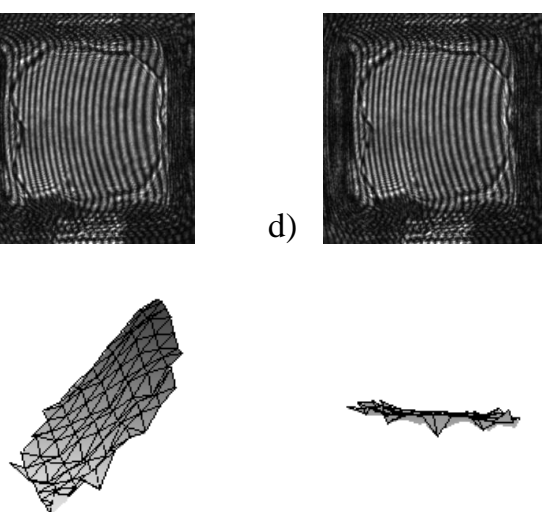
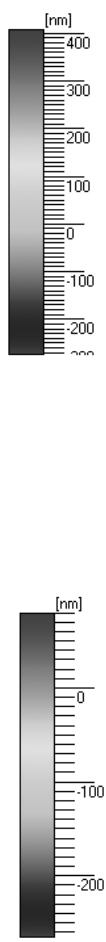

Fig. 10: Interferograms and calculated amplitude distributions of $0.45 \times 0.45 \mathrm{~mm}^{2}$ square membrane obtained for first $(128 \mathrm{kHz})-\mathrm{I}$, and second $(385 \mathrm{kHz})$ - II vibration mode, for a) $0^{\circ}$, b) $90^{\circ}$, c) $180^{\circ}$, d) $270^{\circ}$ of phase difference between excitation and diode controlling signals. Amplitude of excitation signal $\mathrm{U}=10 \mathrm{~V}$, duration of laser pulse $\delta \mathrm{t}=100 \mathrm{~ns}$.
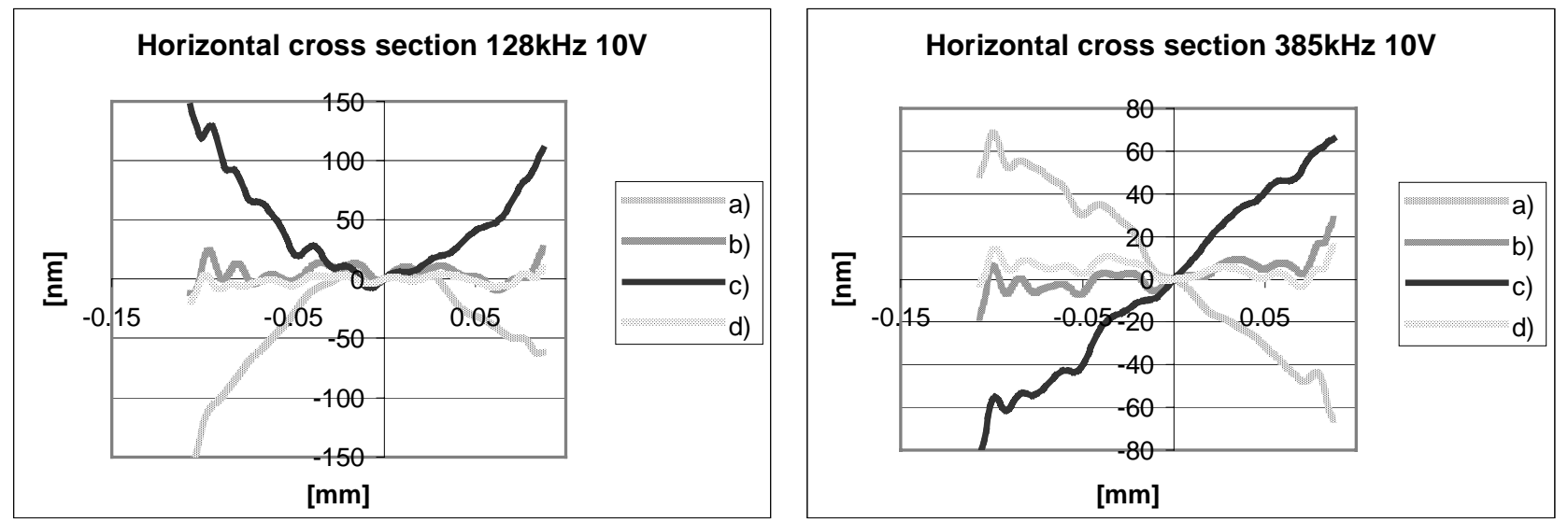

Fig. 11: Horizontal cross sections through vibration amplitude distributions for first (128 kHz) and second (385 kHz) resonance mode of $0.45 \times 0.45 \mathrm{~mm}^{2}$ square membrane obtained for a) $0^{\circ}$, b) $90^{\circ}$, c) $180^{\circ}$, d) $270^{\circ}$ of phase difference between excitation and diode controlling signals. 
The exemplary results of measurements represented by interferograms and calculated amplitude distributions obtained for first and second resonance vibration mode of $0.45 \times 0.45 \mathrm{~mm}^{2}$ square membrane are presented in Fig. 10. Amplitude distributions have been obtained by subtraction of the shape of vibrating membrane from shape of membrane in static state.

The central horizontal cross sections of amplitude distributions are shown in Fig. 11.

Additionally we present the initial approach to find relationship between initial deformation value (see Fig. 8 and Tab. 1) and vibration amplitude value. So the averaged values of vibration amplitude of first resonance mode were calculated in similar way as initial deformation values. Results of that dependence are shown in Fig. 12.
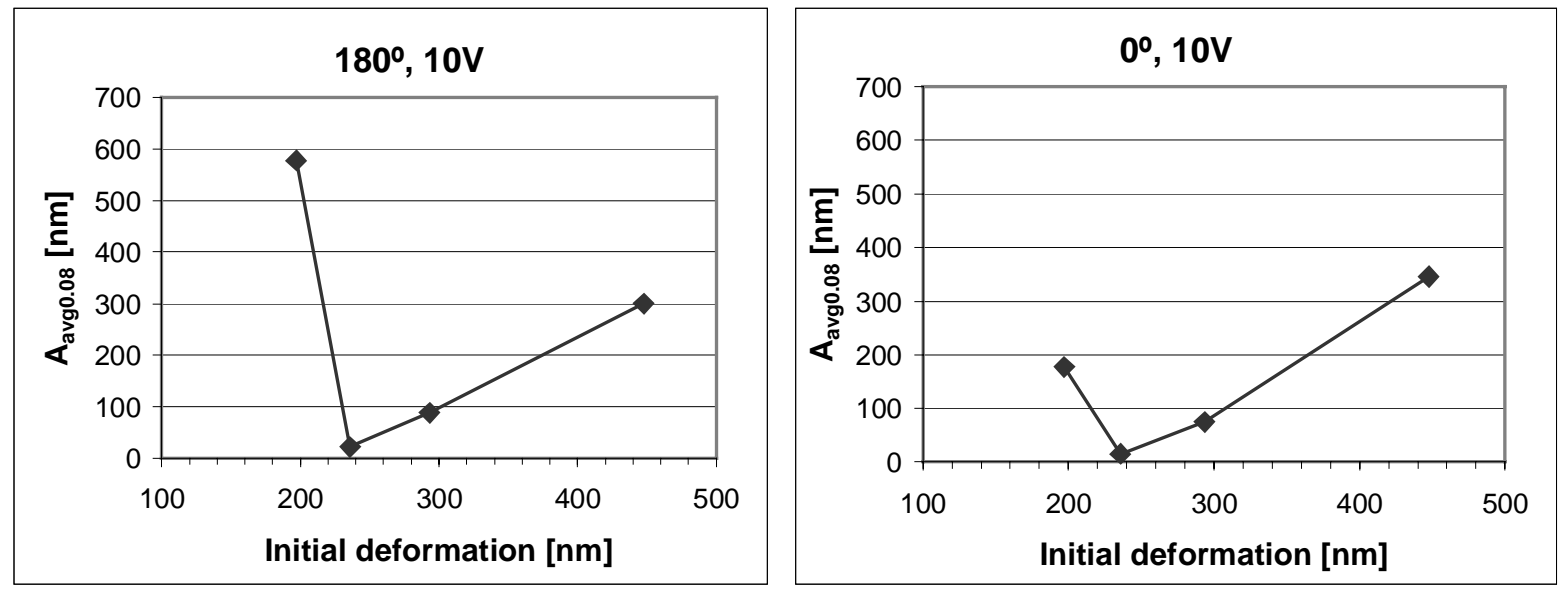

Fig. 12: Average vibration amplitude of first resonance mode as a function of initial deformation of $0.45 \mathrm{x} 0.45 \mathrm{~mm}^{2}$ membranes.

In future this comparison should be performed at the bigger population of the membranes as it seems that such analysis may be excellent source of information for optimization of parameters of technological processes during sequential stages of micromembranes manufacturing.

\section{CONCLUSIONS}

The results of measurements and analysis of methodology applied show that stroboscopic interferometric method is the best solution for quantitative analysis of vibrating microelements. It has to be supported by the enhanced time-average interferometric method and phase shifting interferometry applied for static elements. The extended statistical and comparison analysis performed on the data obtained at static and dynamic modes may provide additional criteria and indications for optimization of parameters of manufacturing process.

\section{REFERENCES}

[1] M. Kujawinska, C. Gorecki, "New challenges and approaches to interferometric MEMS and MOEMS testing", Proc. SPIE, v. 4900, pp. 809-823, 2002.

[2] S. Petitgrand, R. Yahiaoui, A. Bosseboeuf, K. Danaie, "Quantitative time-averaged microscopic interferometry for micromechanical device vibration mode characterization”, Proc. SPIE, v. 4400, pp. 51-60, 2001.

[3] L. Salbut, K. Patorski, M. Jozwik, J. Kacperski, C. Gorecki, A. Jacobelli, T. Dean, "Active micro-elements testing by interferometry using time-average and quasi-stroboscopic techniques”, Proc. SPIE, v. 5145, pp. 23-32, 2003.

[4] S. Petitgrand, R. Yahiaoui, K. Danaie, A. Bosseboeuf, J.P. Gilles, “3D measurement of micromechanical devices vibration mode shapes with a stroboscopic interferometric microscope", Optics and Lasers in Engineering, 36, pp. 77-101, 2001. 